\title{
À propos de l'abolition du régime seigneurial
}

\section{Georges Baillargeon}

Volume 22, numéro 3, décembre 1968

URI : https://id.erudit.org/iderudit/302796ar

DOI : https://doi.org/10.7202/302796ar

Aller au sommaire du numéro

Éditeur(s)

Institut d'histoire de l'Amérique française

ISSN

0035-2357 (imprimé)

1492-1383 (numérique)

Découvrir la revue

Citer cet article

Baillargeon, G. (1968). À propos de l'abolition du régime seigneurial. Revue d'histoire de l'Amérique française, 22(3), 365-391.

https://doi.org/10.7202/302796ar d'utilisation que vous pouvez consulter en ligne.

https://apropos.erudit.org/fr/usagers/politique-dutilisation/ 


\section{À PROPOS DE L'ABOLITION DU RÉGIME SEIGNEURIAL}

\section{Le régime seigneurial a-t-il été réformé ou aboli?}

Dans son Histoire économique, M. Fernand Ouellet prétend qu'en 1854 le régime seigneurial n'a pas été aboli. ${ }^{1}$ Or, dans un article écrit par le même auteur en 1955, le régime seigneurial avait été aboli. ${ }^{2}$ Dans son Histoire économique elle-même, on trouve la phrase suivante: "L'abolition du régime seigneurial ne faisait pas partie d'une offensive destinée à déposséder les Canadiens français d'une institution indispensable à leur survie nationale; elle faisait partie d'un programme de réformes dicté par des considérations économiques et sociales." 3 N'importe quelle personne qui lit ces lignes en conclut que le régime seigneurial a été aboli, sinon l'auteur aurait écrit: Le projet d'abolition ... ou Le plan visant à l'abolition ... ou L'idée d'abolir... etc. Mais, même sans ce passage, la contradiction est flagrante. $M$. Ouellet aurait bien fait de s'en tenir à ce qu'il avait écrit en 1955, car, même si on devine ce que l'auteur veut dire, son affirmation “il n'y aura en 1854 qu'une réforme et non une abolition de la seigneurie" est insoutenable au point de vue juridique. La loi de 1854, intitulée "Acte concernant l'abolition générale des droits et devoirs féodaux", est catégorique sur ce

1 Voici ses propres mots: "il n'y aura en 1854 qu'une réforme et non une abolition de la seigneurie." Et plus loin, il répète: "La seigneurie, telle que réformée par cette législation, etc.". V. Histoire économique et sociale du Québec, 1760-1850, 467.

$2 \mathrm{M}$. Ouellet écrivait alors: "L'abolition du régime seigneurial marquait une étape importante dans l'histoire canadienne. (...) En libérant la propriété de toutes les entraves posées par le régime seigneurial... L'abolition du régime seigneurial signifiait la victoire du capitalisme... Sur le plan juridique, l'abolition du régime seigneurial impliquait... l'esprit qui avait animé ce régime... n'a pas disparu avec l'abolition de la tenure seigneuriale..." Et, au début (p. 22), il avait écrit: "pendant ces quatorze années qui marquent la fin de la tenure seigneuriale..." V. F. Ouellet, "L'abolition du régime seigneurial et l'idée de propriété", Hermès, vol. 4, no 2 (janv. 1955), 14: 34-36, 22.

3 Histoire économique, 357. 
point. ${ }^{4}$ On ne peut tout de même pas se permettre de faire des erreurs juridiques pour le seul plaisir de créer du sensationnel en histoire. Il y a abolition et abolition, d'accord. On aurait pu avoir une abolition sans dédommagement, on a eu une abolition avec indemnisation, mais abolition aussi réelle que s'il y avait eu confiscation. La loi d'abolition de 1854 a vraiment aboli tous les droits seigneuriaux. Elle les a abolis, nous le répétons, non pas en confisquant la propriété, mais en imposant au bénéficiaire de cet avantage (le censitaire) l'obligation de verser une indemnité à celui qu'on dépouillait de ses droits (le seigneur). Cette indemnité, fixée par le gouvernement, s'appelait la rente constituée, mais ce n'était ni une rente seigneuriale, ni un droit seigneurial, c'était la somme d'argent substituée à tous les droits

4 Le chapitre 41 des Statuts refondus de 1861 commence par ces mots: "Considérant qu'il est expédient d'abolir tous droits et devoirs féodaux... et d'assurer ... une compensation... pour tout droit... qu'il perdra par l'abolition des dits droits; et attendu qu'en vue des grands avantages qui doivent résulter... de l'abolition des dits droits et devoirs féodaux, et de la substitution d'une tenure libre à celle sous laquelle ont été tenues jusqu'ici les propriétés..." etc. L'article 30 de cette loi dit: "Le, depuis et après le jour de la publication dans la Gazette du Canada, ou autre gazette officielle, de l'avis que le cadastre d'une seigneurie a été déposé comme susdit, tout censitaire de la dite seigneurie possédera, ... son fonds en franc-alleu roturier, libre et franc de tous cens, droit de banalité, droit de retrait, et autres droits et charges féodales et seigneuriales de quelqu'espèce que ce soit, excepté la rente constituée qui sera substituée à tous droits et charges seigneuriales; (...) Et le seigneur, comme tel, ne sera après tel temps sujet à aucune obligation onéreuse envers ses censitaires et ne pourra prétendre à aucuns droits honorifiques; et nulle terre ou fonds ne sera à l'avenir concédé par un seigneur pour être tenu autrement que sous la tenure en franc-alleu roturier, ou être sujet à des droits de mutation ou autres redevances féodales." L'article 33 de la même loi dit: "Et depuis et après la publication de tel avis, à l'égard d'une seigneurie en particulier, chaque censitaire, y résidant, tiendra, en vertu d'icelui, sa terre en franc-alleu roturier, quitte et nette de tous droits et redevances féodales et seigneuriales, excepté de la rente constituée substituée aux cens et rentes; et le seigneur tiendra ensuite son domaine, et les terres non concédées dans telle seigneurie, et tous pouvoirs d'eau et immeubles lui appartenant alors, en franc-alleu roturier..." Enfin, l'article 91, qui résume l'esprit de cette loi, dit: "l'objet de cet acte... est déclaré être: d'abolir, aussitôt que possible, tous droits, charges et redevances féodales et seigneuriales, en leur substituant des rentes constituées d'égale valeur, - d'accorder au seigneur une indemnité raisonnable, et rien de plus, pour tous les droits lucratifs que la loi lui donne, et que cet acte abolira, - et d'aider le censitaire à même les fonds provinciaux à racheter ces charges seigneuriales qui sont si préjudiciables à son indépendance, à son industrie et à son esprit d'entreprise..." Comment, devant des textes de loi aussi catégoriques peut-on affirmer que le régime seigneurial n'a pas été aboli ? 
seigneuriaux abolis. C'est cette rente constituée que les ex-censitaires étaient libres de racheter à leur gré. Et, contrairement aux apparences, substituer une indemnité fixe aux droits seigneuriaux abolis n'était pas remplacer bonnet blanc par blanc bonnet. En premier lieu, on donnait aux censitaires la chance de se débarrasser de ce fardeau, ce qu'ils n'auraient jamais pu faire sans cette loi, car les droits qu'on abolissait étaient, de leur nature, des droits perpétuels, non rachetables, dont le censitaire ne pouvait pas, avant la loi de 1854 , se libérer, même s'il le voulait, même s'il en avait les moyens. Le second avantage procuré au censitaire consistait en ce qu'on figeait, ou fixait de façon à ce qu'elle ne puisse pas augmenter l'indemnité due aux seigneurs pour des droits casuels qui, en certains cas (tels les lods et ventes) auraient pu lui rapporter dix fois plus en une seule année. Cela ne veut pas dire que la loi de 1854 a été la meilleure loi imaginable dans les circonstances. Mais, ce que ceux qui critiquent cette loi ne savent pas, c'est qu'on a aboli le régime seigneurial non pas comme on a voulu, mais comme on a pu. ${ }^{5}$

Si maintenant on compare entre elles les différentes parties du paragraphe où $M$. Ouellet dit qu'il n'y a pas eu d'abolition, c'est mieux encore. Dans ce paragraphe, il dit 1) qu'en 1854 on "retranche du régime tout ce qui nuit... au capitalisme... et tout ce qui heurte les censitaires"; 2) mais que, "quant aux cens et rentes et aux droits afférents, les tenanciers sont libres de les racheter ou non." ${ }^{6}$ Relativement à ce paragraphe, voici quelques questions que nous nous posons. 1ère question: Si on a retranché tout ce qui nuit... et tout ce qui heurte, comment se fait-il que les tenanciers aient, après cela, à racheter les cens et rentes et les droits que l'auteur appelle afférents ? 2e question: S'il reste

${ }^{5} \mathrm{G}$. Baillargeon, "La tenure seigneuriale a-t-elle été abolie par suite des plaintes des censitaires ?", Revue d'Histoire de l'Amérique française, 21-1 (juin 1967) : 76-79.

$6 \mathrm{M}$. Ouellet écrit: "Ainsi la solution finale de la question seigneuriale sera... il n'y aura en 1854 qu'une réforme et non une abolition de la seigneurie.... on retranche du régime tout ce qui nuit directement au capitalisme du moment et tout ce qui heurte les censitaires. (...) Quant aux cens et rentes et aux droits afférents, les tenanciers sont libres de les racheter ou non." Histoire économique, 467. 
à racheter les cens et rentes et les droits, qu'est-ce qu'on a supprimé ? 3e question: Quand l'auteur écrit: "on retranche du régime tout ce qui nuit directement au capitalisme du moment et tout ce qui heurte les censitaires", ce tout ce qui nuit et ce tout ce qui heurte, nous voudrions bien savoir ce que c'est, puisque ce ne sont ni les cens et rentes ni les droits. 4e question: D'après le paragraphe en question, il semblerait que les cens et rentes et les droits que l'auteur appelle afférents ne nuisaient pas au capitalisme, ne heurtaient pas les censitaires. Alors, pourquoi dans les pages précédentes tant d'insistance à dire qu'il fallait abolir le régime seigneurial ?

Pour exprimer toute notre pensée et pour replacer les choses à l'endroit, disons que si vraiment - comme l'affirme M. Ouellet - la loi avait laissé les censitaires libres de racheter ou non les droits des seigneurs, M. Ouellet aurait eu raison de dire que le régime seigneurial n'avait pas été aboli, mais que, malheureusement, il s'est fourvoyé en disant que la loi de 1854 avait laissé les censitaires libres de racheter ou non les droits seigneuriaux. Si on avait laissé cette liberté aux censitaires, un très grand nombre d'entre eux ne les auraient pas rachetés à ce jour et nous serions encore sous le régime seigneurial. Mais justement, on n'a pas laissé cette liberté au censitaire, on n'a laissé subsister aucun droit seigneurial, on les a tous abolis. La seule liberté qu'on ait laissée à l'ex-censitaire, a été celle de différer le paiement de l'indemnité due à son ex-seigneur en échange de l'abolition de tous ses anciens droits. Et si l'excensitaire se prévalait de cette liberté de différer le paiement de la somme due comme indemnité, il se trouvait dans la situation de tous ceux qui doivent de l'argent, de tous ceux qui empruntent de l'argent, il devenait un débiteur et il devait verser un intérêt annuel de $6 \%$ à celui à qui il devait cet argent (son créancier). C'est ce qu'on appelait la rente constituée, mais ce n'était pas un droit seigneurial, car n'importe qui pouvait faire des constitutions de rente.

En outre, dans ce passage, M. Ouellet dit que 1) 1854 n'a pas apporté l'abolition de la seigneurie; 2) 1854 n'a apporté qu'une 
réforme de la seigneurie; 3) 1854 a apporté "la solution finale de la question seigneuriale". ${ }^{7}$ En bonne logique, vous concluez que nous sommes encore (puisque la solution était finale) sous le régime de la seigneurie réformée, mais non abolie. Hé bien, vous vous trompez, car $\mathbf{M}$. Ouellet termine son paragraphe en disant que nous sommes sous la tenue de franc-alleu roturier. ${ }^{8}$ La solution finale n'avait pourtant pas amené, d'après M. Ouellet, d'abolition du régime seigneurial mais seulement une réforme et pourtant nous sommes aujourd'hui sous la tenure franc-alleu roturier. Comprenne qui pourra.

\section{Le franc-alleu}

Depuis l'entrée en vigueur de la loi d'abolition du régime seigneurial de 1854, les terres des anciennes seigneuries sont tenues en franc-alleu roturier. Or, l'alleu ou le franc-alleu n'a rien à voir avec le régime seigneurial. Alleu vient de allod, qui signifie: propriété complète. Il se définit: "Propriété libre de tous droits seigneuriaux." Vestige de l'ancienne propriété romaine, l'alleu ou franc-alleu, loin d'être une forme de tenure seigneuriale, était justement une exception au régime seigneurial, l'opposé du régime seigneurial. L'alleu ou franc-alleu était "un domaine possédé en libre propriété sans qu'aucun lien de subordination" n'attachât l'alleutier à celui qui lui avait cédé l'alleu.

\section{Franc-alleu et soccage}

Il faudrait encore relever l'opinion selon laquelle le francalleu roturier ressemblait seulement quelque peu au franc et commun soccage. ${ }^{\ominus}$ Nous aimerions que l'auteur nous révèle les

$7 \mathrm{~V}$. ci-devant, note 6.

8 Voici comment M. Ouellet termine son paragraphe: "La seigneurie, telle que réformée par cette législation, se rapprochait quelque peu du système qui prévalait dans les cantons. En effet, les différences n'étaient pas très considérables entre le franc aleu roturier et le franc et commun soccage." Voilà. Vous avez vu le tour de passe-passe ? A l'avant-dernière phrase nous sommes encore sous le régime de la seigneurie, à la dernière phrase nous en sommes sortis et nous sommes sous la tenure de franc-alleu roturier ! Et le tour est joué. V. Histoire économique, 467.

9 V. ci-devant, note 8. 
avantages qu'il y a à posséder une terre en franc et commun soccage dans les Cantons de l'Est plutôt que d'en posséder une en franc-alleu roturier dans une autre partie de la Province. Le franc et commun soccage tel qu'il existait dans les Cantons de l'Est et le franc-alleu roturier introduit pour remplacer la tenure en censive sont absolument équivalents. Seul le système de lois que le gouvernement impérial déclara en vigueur dans les Cantons, différait du système de lois en usage dans le reste de la Province. Mais, système de lois n'a jamais été synonyme de tenure.

\section{Franc-alleu et tenure seigneuriale}

De plus, notons que des deux dernières phrases du paragraphe en question, il ressort que, pour M. Ouellet, seigneurie réformée est synonyme de franc-alleu roturier, ${ }^{10}$ ce qui est insoutenable, comme nous l'avons expliqué plus haut. Le franc-alleu roturier n'est ni de la seigneurie, ni de la seigneurie réformée, parce que le mot seigneurie suppose un seigneur et que le francalleu roturier se caractérise justement par l'absence de seigneur.

\section{Aucun "amendement du système seigneurial"}

Pendant que nous en sommes à cette question de réforme et d'abolition, signalons que, d'après $M$. Ouellet, il y a eu, dans la "décennie 1840-50" un "amendement du système seigneurial"."11 Mais, chose étrange, l'auteur n'en révèle pas la teneur. Il ne parle que de la loi de 1854, qui a pour titre "Acte concernant l'abolition générale des droits et devoirs féodaux", qui serait un drôle d" "amendement" et qui se logerait d'une façon assez bizarre dans la décennie 1840-50. A moins de dire qu'amendement est synonyme de suppression, de dire qu'extraire une dent c'est la guérir, il faut dire que, bien qu'il y ait eu, sous l'adminis-

$10 \mathrm{~V}$. ci-devant, note 8.

11 Dans le chapitre consacré à la période 1840-1850, après avoir bien spécifié ( $p$. 441) qu'il parle de "la décennie 1840-1850" en ces termes: "la décennie 1840-50 est marquée par une accumulation sans précédent de réformes...", M. Ouellet cite comme exemple de ces réformes (p. 442) qu'il y a eu "l'amendement du système seigneurial". V. Histoire économique, 441,442 . 
tration anglaise, diverses lois autorisant le rachat total ou partiel des droits seigneuriaux, il n'y a eu, ni dans la décennie 1840-50, ni dans la décennie suivante, ni en aucun temps après 1760 , aucune loi de réforme ou d'amendement du régime seigneurial, tous les projets en ce sens ayant été bloqués par le Conseil législatif ou quelque autre autorité.

\section{Les droits "afférents"}

Toujours au sujet du même paragraphe, il faut aussi mentionner que M. Ouellet a une façon de diviser les droits seigneuriaux qui est loin d'aider à la compréhension du texte. Il les divise en cens et rentes d'une part, et en droits afférents d'autre part. ${ }^{12}$ Comme le mot afférent veut dire: "qui revient à chacun ou à quelqu'un", et que, d'autre part, les règles de la logique exigent que les parties d'une division s'opposent entre elles, on voit où cela mène. Il faut probablement entendre qu'il $y$ a des droits qui reviennent à chacun ou à quelqu'un, et d'autres droits - appelés cens et rentes - qui ne reviennent... à personne ! Les droits seigneuriaux étaient, de par leur nature, soit fixes (tels les cens et rentes), soit variables ou casuels (tels les lods et ventes, la banalité, etc.). L'un ou l'autre de ces deux derniers adjectifs aurait été beaucoup plus clair que le mot afférents.

\section{Attitude de "l'élite" et des paysans}

M. Ouellet soutient en premier lieu que c'est "une certaine élite" qui "défend vigoureusement la seigneurie" et le paysan qui commence à être opposé à ce système. ${ }^{13}$ Pour nous convaincre de ce qu'il vient de dire, l'auteur cite un témoignage de Boucher de la Bruère contre le régime seigneurial. ${ }^{14}$ Or, Boucher de la

12 Voici ses propres mots: "Quant aux cens et rentes et aux droits afférents, les tenanciers sont libres de les racheter ou non." V. Histoire économique, 467.

13 Voici les mots mêmes de M. Ouellet: "A partir de 1840, même si une certaine élite intéressée ou, encore, fixée aux perceptions passées défend vigoureusement la seigneurie, l'attitude de l'habitant a tendance à se modifier en conséquence d'une prise de conscience." V. Histoire économique, 462.

14 "Le travail et le capital, ces deux principes de tout progrès matériel. se refusent à prêter leur secours à des entreprises dont les profits seront partagés par une classe privilégiée qui ne fournira ni travail ni capital." V. Histoire économique, 463. 
Bruère est un fils de seigneur, il fait partie de la classe riche et instruite, de l'élite par conséquent et il parle contre le régime seigneurial ! On voit que ce témoignage est fort pour nous convaincre que l'élite défend le régime et que "l'attitude de l'habitant a tendance à se modifier". Donc, la citation tend à prouver exactement le contraire de ce que l'auteur affirme. Peu importe, les lecteurs penseront que Boucher de la Bruère est un pauvre cultivateur ! D'ailleurs, ça n'a pas d'importance, puisque deux pages plus loin, M. Ouellet écrit exactement le contraire de ce qu'il vient d'affirmer. Il écrit maintenant: "le discrédit, qui pèse sur cette tenure parmi une certaine élite, a aussi gagné le milieu paysan".15 Donc, à la page 462, l'auteur dit qu' "une certaine élite ... défend ... la seigneurie", alors qu'à la page 464 "le discrédit... pèse sur cette tenure parmi une certaine élite". De plus, à la page 462 c'est le paysan qui a "une prise de conscience", qui modifie son attitude, pendant que l'élite défend la seigneurie; alors qu'à la page 464 c'est parmi l'élite que cette tenure est tombée en discrédit et que le mouvement "a ensuite gagné le milieu paysan" !

\section{A-t-on maintenu l'intégrité du régime?}

M. Ouellet écrit qu'avant 1854 on avait préservé l'intégrité du régime seigneurial. ${ }^{16}$ Personnellement, nous aurions omis le mot intégrité. En effet, on peut soutenir que le régime seigneurial a évolué après 1760 par suite des décisions des Cours de justice substituées à celle de l'intendant, attendu que nombre de juges étaient soit des seigneurs, qui avaient des intérêts à préserver, soit des gens d'origine britannique, qui s'inspiraient de la tradition anglaise plutôt que de la coutume française et des décisions des intendants. Mais ce n'est pas sur ce point que nous voulons insister.

15 Histoire économique, 464.

16 Voici ses propres termes: "Pendant longtemps, clercs, seigneurs canadiens-français et professions libérales avaient pu maintenir l'intégrité du régime en représentant les abolitionistes comme des ennemis de la nation et de ses droits. La paysannerie avait accepté cette vue des choses et, en même temps, accepté comme un moindre mal les abus des seigneurs." V. Histoire économique, 462. 


\section{A-t-on maintenu le régime?}

Si on n'attache pas d'importance au mot intégrité, la phrase devient: "Pendant longtemps, clercs, seigneurs canadiensfrançais et [membres des] professions libérales avaient $p u$ maintenir... [le] régime". ${ }^{17}$ Idée exprimée ailleurs sous une autre forme: "Le système [seigneurial] ne se perpétue que grâce aux efforts des [membres des] professions libérales."18 C'est se donner bien du mal pour trouver une cause externe à un phénomène qui n'en avait pas besoin. Personne n'a maintenu le régime seigneurial; jamais on n'a eu d'efforts à faire pour le maintenir. Il s'est maintenu tout seul, par le seul fait que personne ne trouvait la façon de l'abolir qui n'aurait présenté aucun inconvénient et parce que les seigneurs d'origine britannique qui, d'après M. Ouellet, voulaient l'abolition du régime seigneurial (puisqu'il spécifie que ce sont les seigneurs canadiensfrançais qui ont maintenu le régime seigneurial), parce que, disions-nous, les seigneurs d'origine britannique, sauf un, ne se sont pas prévalus des lois de 1822 et de 1825 qui les autorisaient à mettre la hache au régime seigneurial dans leurs seigneuries, et parce que, dans cette unique seigneurie où on pouvait se libérer, les Britanniques - comme les Canadiens français se sont contentés de parler contre le régime seigneurial sans qu'aucun n'agisse pour se libérer. Ce n'est pas un Canadien français qui l'a dit, c'est un Britannique, l'agent même de cette seigneurie, Edward Gibbon Wakefield: "Grand nombre de censitaires des deux origines, mais surtout les Censitaires Écossais, font sonner bien haut leur désir de commuer; mais je n'en ai pas trouvé un seul" qui ait consenti à racheter les droits dus au seigneur. ${ }^{19}$ Cela prouve qu'en bien des cas - nous ne disons pas dans tous - les Britanniques ne parlaient contre les droits seigneuriaux que parce qu'ils croyaient pouvoir en obtenir l'abolition sans avoir à payer d'indemnité. Longtemps nous nous sommes laissé berner par les déclarations des Britanniques con-

17 Histoire économique, 462.

18 Ibid., 351.

19 No 69, "Opinion d'Edward Gibbon Wakefield", 2 sept. 1842, Rapport des Commissaires, 220. 
tre le régime seigneurial, mais ce témoignage de Wakefield nous a ouvert les yeux. Voilà la réalité. Voilà qui prouve que les Canadiens français n'ont pas eu besoin de faire des efforts pour maintenir le régime seigneurial.

\section{Les Canadiens français étaient-ils tous favorables au régime?}

Avant 1830, la majorité des Canadiens français avait été favorable à la tenure seigneuriale, ${ }^{20}$ mais pas seulement par "obstination" ou par "aveuglement paysan". ${ }^{21}$ Puis le vent tourne. Un contemporain a fixé le point tournant vers 1830. Par la suite, vers 1834-35 surtout, il devient évident que les Canadiens français ne sont pas tous en faveur du maintien du régime seigneurial. Les résolutions adoptées lors des assemblées de comtés de 1836-37 abondent en témoignages qui prouvent que les idées ont changé. Puis, les témoignages se multiplient. Il suffit de lire, entre autres, les lettres qui accompagnent le Rapport des Commissaires ${ }^{22}$ de 1843 , pour se rendre compte qu'il y a des prêtres, des seigneurs canadiens-français, des notaires et des arpenteurs canadiens-français qui recommandent l'abolition du régime seigneurial. L'abbé Chiniquy, dont les idées ne sont pas contenues dans ce Rapport, mais que nous citerons plus loin, en était un autre qui prônait l'abolition du régime seigneurial.

\section{Les seigneurs d'origine britannique voulaient-ils l'abolition?}

La ligne de démarcation tracée par M. Ouellet entre les seigneurs canadiens-français et ceux d'origine britannique, et selon laquelle les seigneurs canadiens-français auraient travaillé à conserver leurs droits de seigneurs alors que ceux

20 Les Canadiens veulent conserver le régime seigneurial 1800-1826", Revue d'Histoire de l'Amérique française, 7-1, (juin 1953) : 45.

21 Histoire économique, 351.

22 Rapport des Commissaires nommés pour s'enquérir de l'état des lois et autres circonstances qui se rattachent à la tenure seigneuriale dans le Bas-Canada, et appendice (Montréal, 1844). Ce livre se trouve textuellement, sauf la pagination, dans l'appendice du troisième volume des Journaux de l'Assemblée législative de la province du Canada, 1843, 1ère partie, sous la lettre F. 
d'origine britannique en auraient voulu l'abolition, ne correspond pas à la réalité. ${ }^{23}$ Les Anglais étaient opposés au régime seigneurial tant qu'ils n'étaient pas seigneurs; une fois seigneurs, c'était différent ! Le cas d'Ellice ne change rien à cette affirmation. Les seigneurs d'origine britannique qui ont réellement voulu abolir le régime seigneurial dans leurs seigneuries (Caldwell, Ellice), ont travaillé dans ce sens parce qu'ils espéraient faire plus d'argent de cette façon-là. ${ }^{24}$ C'est tout. Ellice l'a d'ailleurs dit en toutes lettres. ${ }^{25}$ S'il est vrai que les Anglais sans distinction voulaient l'abolition du régime seigneurial, pourquoi un si grand nombre d'entre eux achetaient-ils des seigneuries ? M. Ouellet note lui-même ce fait lorsqu'il écrit: "Cet inventaire permet de saisir l'intérêt porté par les Britanniques à l'acquisition de seigneuries." ${ }^{26} \mathrm{Ils}$ achetaient ces seigneuries pour y abolir le régime seigneurial je suppose ? En ce cas, pourquoi les Anglais qui possédaient des seigneuries et qui étaient des capitalistes et qui auraient dû, d'après ce qu'affirme M. Ouellet, être opposés au régime seigneurial - tel John Pangman, fils d'un bourgeois de la Compagnie du Nord-Ouest et seigneur de Lachenaie ne se sont-ils pas prévalus (sauf un) des lois de 1822 et de 1825 pour faire changer la tenure de ces seigneuries ? Parce qu'ils étaient opposés au régime seigneurial ou parce qu'ils étaient, en tant que capitalistes, là où il y avait de l'argent à faire, c'està-dire à la tête d'une seigneurie ? Pourquoi en 1824 James McCallum prit-il la peine de faire ériger en seigneuries sous les noms de Saint-Jacques et Saint-Normand, des terres $q u$ 'il possédait déjà en franc et commun soccage ? Pourquoi Jacob Moun-

23 Tant dans le passage de la page 462 cité à la note 15 , que dans celui de la page 351 où il parle de "l'obstination des seigneurs de vieille appartenance". Notons que l'expression "seigneurs de vieille appartenance" pourrait prêter à confusion, attendu que, dans les écrits des Britanniques, l'expression "seigneurs anciens sujets" désigne les Britanniques. Nous supposons qu'il s'agit des Canadiens français à cause du mot obstination et parce que, dans le contexte, les blâmes sont pour les Canadiens français.

24 "Les Canadiens veulent conserver le régime seigneurial 1800-1826", Revue d'Histoire de l'Amérique française, 7-2, sept. 1953: 227-235.

25 Témoignage d'Edward Ellice, 15 mai 1828, Appendice du XXXVIIIe volume des Journaux de la Chambre d'Assemblée du Bas-Canada, 2, [Québec, 1829], Appendice HH, [n.p.].

26 Histoire économique, $\mathrm{XXV}$. 
tain fit-il ériger en seigneurie sous le nom de Thwaite les terres qu'il possédait également déjà en franc et commun soccage ? ${ }^{27}$ N'est-ce pas une étrange façon de vouloir l'abolition du régime seigneurial ? La démarcation ne se serait-elle pas faite beaucoup plus selon la ligne de l'intérêt que selon celle de l'origine raciale ? La vérité, c'est qu'il y a eu des seigneurs canadiens-français qui ont prôné l'abolition du régime seigneurial, comme il y a eu des seigneurs de langue anglaise qui ont été opposés à tout projet de loi visant à abolir ce régime. En fait, les pires opposants à tous les projets de loi visant à mettre fin au régime seigneurial ont été des seigneurs de langue anglaise.

\section{Les abus des seigneurs, un moindre mal}

Quand M. Ouellet écrit que "la paysannerie avait ... accepté comme un moindre mal les abus des seigneurs", 28 il oublie ce qui se passait à cette époque dans les Cantons de l'Est, ${ }^{29}$ autrement il aurait écrit: les abus des seigneurs étaient un moindre mal. Cela a été dit (entre autres) non par un "clerc", ni par un "seigneur canadiens-français", mais par John Neilson en ces termes: "L'une ou l'autre tenure est bonne, si l'on en exclut les abus qui règnent dans les deux, mais beaucoup plus, je crois, dans la tenure en soccage. ${ }^{30}$ Cet Ecossais de bon jugement avait pris la défense des Canadiens français devant un comité des Communes britanniques en 1828. Lorsqu'on lui avait demandé:

Les habitans du Bas-Canada qui descendent des colons primitifs, ne préfèrent-ils pas de beaucoup la tenure sous laquelle sont les terres dans les seigneuries, à celle du franc et commun soccage ?

${ }^{27}$ I. Caron, La Colonisation de la province de Québec. Les Cantons de l'Est 1791-1815, 176; Continuation de l'Appendice du XLIIe volume des Journaux de la Chambre d'Assemblée de la province du Bas-Canada, Appendice NN, 54, 55, 64; P.-G. Roy, Toutes petites choses du régime anglais, $1: 44$.

28 V. ci-devant, note 15.

29 "Les Canadiens veulent conserver le régime seigneurial 1800-1826", Revue d'Histoire de l'A mérique française, 7-3, (déc. 1953) : 359.

30 Témoignage de John Neilson, 31 déc. 1823, Appendice du XXXIIIe volume des Journaux de la Chambre d'Assemblée de la Province du BasCanada, Appendice R, Appendice A. 
John Neilson (rappelons qu'il était avocat) avait répondu:

En réalité, ils ne s'occupent pas beaucoup de la tenure. Ce qu'ils veulent, c'est d'avoir des terres à bon marché, et de les avoir aisément et sans beaucoup de dépense. Ils ne se tromperont jamais sur ce point; ce qui sera le moins cher et le plus facile sera ce qu'ils aimeront le mieux.

C'est étrange comme cette opinion diffère de celle de M. Ouellet, pour qui le choix des Canadiens français est dû à "l'aveuglement paysan". ${ }^{31}$ Puis, le comité insistant pour savoir si les Canadiens français ne faisaient vraiment pas de différence entre les deux tenures, Neilson avait complété sa pensée comme suit:

Le bruit qu'on a fait au sujet des tenures a été déclenché par la tentative de changer les lois du pays en même temps qu'on essayait de changer les tenures. Or les lois qui régissent les propriétés particulières, qui règlent l'héritage des enfants, et tout le reste, ont toujours été chères à tous les peuples; il faut que ce soient de bien mauvaises lois pour que le peuple ne s'attache pas à celles sous lesquelles il a vécu pendant un temps considérable, et sous lesquelles il a possédé ses propriétés avec sureté. Du moment qu'on a parlé de changer les lois, on a excité l'alarme dans tout le pays. La même chose se produirait si on parlait de changer les lois qui régissent les propriétés en Angleterre ou en Ecosse. ${ }^{32}$

Voilà une façon sensée d'exposer le problème. Il faudrait développer ce thème et brosser le tableau au complet pour faire tomber cette réplique que quelques-uns ont déjà sur le bout de la langue, mais cela nous mènerait trop loin. Nous le ferons ailleurs, ne craignez rien. Enfin, ajoutons un témoignage d'un des représentants canadiens-français des professions libérales, considérés par M. Ouellet comme des arriérés, ${ }^{33}$ Denis-Benjamin Viger, qui, déjà en 1831, était obligé de réfuter ceux chez qui

31 Histoire économique, 351.

32 Témoignage de John Neilson, 24 mai 1828, Appendice dw XXXVIIIe volums des Journaux de la Chambre d'Assemblée du Bas-Canada, 2 Appendice $H H$ [n.p.].

33 V. ci-devant, notes 12 et 15. 
M. Ouellet a puisé ses idées. Denis-Benjamin Viger, accrédité officiellement par l'Assemblée législative du Bas-Canada pour lui servir de porte-parole auprès du gouvernement impérial, représentant par conséquent l'idée d'au moins quelques Canadiens français, écrivait:

Je dois relever ici ce qu'il y a de beaucoup plus que singulier dans une idée mise au jour dans plusieurs des écrits que le Canada a vu éclore... On a dit et répété que les Canadiens poussoient l'Aveuglement de l'attachement à leurs institutions jusqu'à ne vouloir pas entendre parler de prendre du Gouvernement des Concessions de terres allodiales et dégagées de toute redevance, qu'ils avoient une prédilection exclusive pour les terres assujetties à des rentes, lots et ventes et autres droits seigneuriaux ! On devroit croire que c'étoit là sans doute une plaisanterie. Rien n'étoit pourtant plus sérieux que cette assertion et les commentaires dont elle a été souvent accompagnée...

... peut-on supposer que, si le Gouvernement avait mis autant de moyens en œuvre pour attirer les Canadiens vers les terres soccagères qu'il en a pris pour les en détourner, nos gens n'auraient pas préféré s'établir sur des terres libres et dégagées de toute redevance plutôt que sur celles qui en étaient chargées ? C'est réellement s'exposer soi-même et prêter le flanc au ridicule que de conserver un ton de gravité en signalant la bizarrerie de semblables opinions. Ce devient pourtant souvent une nécessité indispensable quand on discute des objets d'intérêt public relativement au Bas-Canada. ${ }^{34}$

Voilà ce que pensait Denis-Benjamin Viger, il y a plus d'un siècle, de l'opinion ramenée à la surface par M. Ouellet.

\section{Les immigrants}

M. Ouellet qui, dans une section de son livre, méprise les défenseurs du régime seigneurial, nous révèle ailleurs que les

34 D.-B. Viger, "Observations relatives à quelques-uns des griefs de l'Assemblée du Bas-Canada énoncés dans son adresse en 1831", 24 sept. 1831, APC, Q 214-1: 68; se trouve aussi à Q 220-3: 509 . 
immigrants britanniques qui demeurent dans le Québec, "choisissent de se fixer dans le territoire seigneurial". ${ }^{53}$ Ça c'est le comble ! L'auteur reproche aux Canadiens français d'être frappés d' "aveuglement paysan", d'être des retardataires, des obstinés, des gens "fixés aux perceptions passées" parce qu'ils préfèrent la tenure seigneuriale au système tel qu'il fonctionne dans les Cantons de l'Est; il laisse entendre que les gens d'origine britannique sont plus intelligents et plus progressifs parce qu'ils veulent l'abolition du régime seigneurial, et, tout bonnement, sans faire le lien entre les deux idées, il vient nous dire que les immigrants britanniques "qui demeurent dans le Bas-Canada ... choisissent de se fixer dans le territoire seigneurial". Mais où est la logique dans tout ça? Pourquoi est-ce de "l'obstination" et de "l'aveuglement paysan" pour les Canadiens français de préférer les abus des seigneurs à ceux qui ont cours dans les Cantons, quand les Britanniques préfèrent eux aussi les abus des seigneurs? Si, comme l'affirme $M$. Ouellet, les immigrants britanniques qui se fixent au Québec choisissent de s'établir dans les seigneuries, c'est qu'ils jugent, eux aussi, que les abus des seigneurs sont encore préférables à ceux qui existent dans les Cantons. De quel droit, alors, blâmer les Canadiens français d'avoir pensé de même ?

\section{Les seigneurs n'étaient pas tous des vampires}

M. Ouellet fait une généralisation injustifiée et injustifiable quand il écrit: "la politique des seigneurs demeure la même qu'autrefois. L'accroissement des cens et rentes, etc." ${ }^{36}$, comme si tous les seigneurs s'étaient rendus coupables des abus qu'il énumère; ce qu'il dit carrément d'ailleurs à une autre page: "Partout des pratiques semblables foisonnent..." ${ }^{37}$ Même si un trop grand nombre de seigneurs se sont montrés durs pour leurs censitaires, c'est de l'exagération que de dire qu'ils se sont tous

35 "De 1825 à 1830, 23\% [des immigrants britanniques] se fixent dans le Québec. (...) Ils choisissent de se fixer dans le territoire seigneurial", v. Histoire économique, 350-351.

36 Histoire économique, 462.

37 Ibid., 353. 
rendus coupables d'abus. Il est évident que ceux qu'on dénonce et qui sont, par le fait même, mis en évidence, sont les extorqueurs. Mais, sans vouloir défendre le régime, il faut tout de même reconnaître qu'un grand nombre de seigneurs, surtout dans les districts de Trois-Rivières et de Québec, s'en étaient tenus aux taux en usage sous l'administration française. Dans les discours prononcés en Chambre pendant les débats qui précédèrent l'abolition du régime seigneurial, certains députés l'affirmèrent carrément. Ainsi, Francis Hincks, qui n'était ni un "clerc", ni un "seigneur canadien-français", disait en Chambre, sans que personne ne le réfute: “... l'on veut punir les censitaires, en les obligeant à une commutation forcée... Comment peut-on forcer ceux qui ne se plaignent point de cette tenure à commuer ? Il y a un grand nombre de seigneuries dans lesquelles les exactions ont été peu nombreuses, et il serait injuste d'imposer ... une commutation à ces personnes qui n'ont point d'intérêt à le faire ..." 38 Pour sa part, Pierre-Joseph-Olivier Chauveau, solliciteur général du Bas-Canada, disait: "On a exagéré les vexations des seigneurs; on les a appelés sans distinction des vampires. (...) $J e$ suis loin de croire que la majorité d'entr'eux mérite ce qu'on en a dit." ${ }^{89}$ Et Joseph Cauchon: "Je suis parfaitement libre et ne ressens aucunement la pression de l'opinion publique sur cette question. Dans le comté que je représente, on ne se plaint pas de la tenure seigneuriale, parce qu'il n'y a aucun abus et que les rentes sont peu élevées." ${ }^{40} \mathrm{Ce}$ dernier témoignage prouve que M. Ouellet a exagéré également lorsqu'il a écrit: "De là ce mécontentement généralisé, attisé par les politiciens, qui gagne la masse des tenanciers. Partout on ressent le besoin d'un changement." ${ }^{41}$ La généralisation est excessive. Ce n'était pas partout qu'on était mécontent des seigneurs, ce n'était pas partout qu'on ressentait le besoin d'un changement. Si on se donne la peine de lire les lettres (pas seulement le Rapport) annexées au Rapport des Commissaires ${ }^{42}$ de 1843 , on se rend compte qu'environ la

38 "Tenure seigneuriale", La Minerve, 25-87 ( 7 mai 1853).

39 Débats dans l'Assemblée législative, sur la tenure seigneuriale, 26.

40 "Tenure seigneuriale", La Minerve, 25-85 (10 mai 1853).

41 Histoire économique, 465.

$42 \mathrm{~V}$. ci-devant, note 21 . 
moitié d'entre elles demandent à conserver le régime seigneurial.

\section{Les seigneurs n'ont pas tous refusé de concéder leurs terres}

Ailleurs encore, la tendance à la généralisation pousse $M$. Ouellet à appliquer à un grand nombre de seigneuries le témoignage qu'il a trouvé relativement à l'une d'entre elles. Il écrit: "Dans la seigneurie de Beauharnois, comme dans toutes les seigneuries où il y avait des terres disponibles, l'agent du seigneur qui n'avait pratiquement pas consenti de concessions de 1810 à 1821, le fait maintenant mais avec beaucoup de circonspection." ${ }^{43}$ Cette phrase veut dire que "dans toutes les seigneuries où il $\mathrm{y}$ avait des terres disponibles, l'agent du seigneur... n'avait pratiquement pas consenti de concessions de 1810 à 1821", ce qui est une exagération. C'est un fait que, devenu seigneur de Beauharnois par la mort de son père en 1804, Edward Ellice avait ordonné à son agent de ne plus concéder à cens et rentes aucune terre de sa seigneurie, espérant que plus tard les tenures seraient changées et qu'alors il pourrait disposer de ses terres de façon plus profitable. ${ }^{4}$ Mais cela ne permet pas de dire que tous les seigneurs propriétaires de terres non concédées ont fait la même chose, entre les deux mêmes dates et qu'après 1821 ils ont modifié un peu leur ligne de conduite.

\section{La campagne pour l'abolition a commencé avant 1840}

Pour M. Ouellet, la campagne pour l'abolition du régime seigneurial commence "après 1840".45 Dommage, car elle était commencée avant ça. Pour justifier sa date de 1840 comme point de départ du mouvement abolitionniste, M. Ouellet croit avoir trouvé une raison pour laquelle les paysans se détournent de la tenure seigneuriale: "au sortir des insurrections les habi-

43 Histoire économique, 352.

44 Témoignage d'Edward Ellice, 15 mai 1828, Appendice du XXXVIIIe volume des Journaux de la Chambre d'Assemblée du Bas-Canada, 2, [Québec, 1829], Appendice $\mathrm{HH}$, [n.p.].

45 M. Ouellet écrit: “En somme, après 1840, capitalistes, petits bourgeois d'allégeance libérale, esprits réalistes de toutes teintes, politiques ou idéologiques, mènent une campagne bien organisée en vue d'obtenir l'abolition. de la « féodalité »." V. Histoire économique, 464. 
tants étaient fort endettés, en particulier vis-à-vis des seigneurs". ${ }^{46}$ La raison semble bien bonne, malheureusement ce n'est pas après les insurrections que le mouvement a commencé, mais avant. Chez les Canadiens français, la grande discussion "Le régime seigneurial est-il intrinsèquement mauvais ou sont-ce les abus des seigneurs qui le rendent tel ? Faut-il réformer le régime seigneurial et le conserver, ou l'abolir ?" commence vers 1830. Dans un grand nombre d'assemblées de comtés en 1836-37 (certaines présidées par des seigneurs parfaitement amis du peuple et respectés de lui), les Canadiens avaient demandé l'abolition du régime seigneurial, et un notaire de Laprairie (que nous citerons plus loin) est allé jusqu'à dire que les rebelles de sa région ont fait la révolution pour abattre les seigneurs, qui étaient leur ruine. ${ }^{47}$ Inutile d'ajouter que les dettes envers les seigneurs en question et leurs pareils dataient d'avant les "Troubles".

\section{Oscillations du mouvement antiseigneurial}

Voici, selon nous, quelles furent, grosso modo, les étapes du cheminement de la pensée antiseigneuriale. 1er temps: Les censitaires se demandent: Faut-il réformer ? Ne vaut-il pas mieux abolir ? 2e temps: Avant et pendant les "Troubles", les censitaires demandent l'abolition des droits seigneuriaux. Pendant les assemblées de comtés, on ne précise pas si cette abolition doit comporter une indemnité aux seigneurs, mais les "Troubles" révèlent qu'on entend par là — du moins dans un très grand nombre de cas - une abolition sans versement d'indemnité aux seigneurs. 3e temps: Ne pouvant obtenir l'abolition sans indemnité aux seigneurs, les censitaires créent, en 1848, l'Association pour la réforme du régime seigneurial, c'est-à-dire pour tenter d'obtenir de la Législature une loi imposant une diminution de certains taux de concession et mettant fin aux abus introduits par divers seigneurs. 4e temps: Devant le refus catégorique de Louis-Hippolyte LaFontaine, ${ }^{48}$ qui répond: "Il ne s'agit pas de

46 Ibid., 44.

47 Nous donnerons plus loin d'autres témoignages sur l'ampleur du mouvement abolitionniste pendant les "Troubles".

48 "Revue Parlementaire", La Minerve, 21-42 (1er févr. 1849). 
réformer et de conserver, mais d'abolir en indemnisant", l'Association, qui change son nom en celui de Convention antiseigneuriale, continue à demander en premier lieu une réforme du régime seigneurial et, pour obtempérer partiellement au désir de LaFontaine, $y$ ajoute, mais à son corps défendant et seulement en second lieu (ce que LaFontaine n'acceptera pas), une demande d'abolition avec indemnité aux seigneurs (1849). De simples censitaires embrassent l'idée de LaFontaine. ${ }^{49}$ Ils avouent que c'est très malheureux que tout le monde ne soit pas d'accord sur le remède. ${ }^{50}$ L'abbé Chiniquy se range du côté de LaFontaine: "L'abolition est une question difficile à résoudre, mais elle est loin d'être insoluble; un ajournement augmentera les difficultés, et n'en fera disparaître aucune. Il est démontré que l'abolition seule aura pour effet de trancher les difficultés de détail, que l'on rencontrera à chaque pas, si l'on s'attache seulement à remanier le système usé de la tenure seigneuriale." ${ }^{51}$ L'Avenir qui, avant 1849 , avait considéré l'abolition de la tenure seigneuriale comme impossible et s'était borné à en demander la réforme, commence à en demander l'abolition. ${ }^{52}$ Puis, les assemblées demandant l'abolition du régime seigneurial se multiplient. ${ }^{53}$ Mais, il y a abolition et abolition. Chose certaine, tout le monde n'était pas prêt à payer pour se débarrasser du régime seigneurial.

Les capitalistes ont-ils mené une campagne antiseigneuriale?

Au dire de M. Ouellet, les capitalistes, entre autres, auraient "mené une campagne bien organisée" en vue d'obtenir l'abolition du régime seigneurial. ${ }^{54}$ Nous formulons de très sérieuses réserves au sujet de cette affirmation. Nous n'opposons pas une 1849).

${ }^{49}$ Un Abolitionniste, "Droits seigneuriaux", La Minerve, 21-43 (5 févr.

50 Rouville, "Tenure seigneuriale", La Minerve, 21-49 (26 févr. 1849).

51 Un abolitionniste, "Droits seigneuriaux", La Minerve, 21-62 (12 avr. 1849).

62 F. André-Julien, Les Textes essentiels du journal l' “Avenir" 1847-

1858, (Manuscrit, Univ. de Montréal), 158-159.

c3 "Tenure seigneuriale", La Minerve, 22-10 (11 nov. 1849).

$54 \mathrm{~V}$. ci-devant, note 45 . 
fin de non-recevoir, mais nous ne pouvons pas l'accepter sans preuves. S'il y a eu des capitalistes, nous aimerions les connaître, car les documents que nous connaissons, nous, prouvent le contraire. D'après ces documents, ceux qui se sont débattus, pour améliorer leur sort, ce sont les cultivateurs - la "paysannerie" comme dit M. Ouellet, ceux qu'il accuse d'avoir été frappés d' "aveuglement paysan" - avec l'aide de membres des professions libérales, dont quelques-uns étaient députés à la Législature. Qu'il y ait eu quelques capitalistes qui les aient aidés, c'est dans le domaine du possible, mais certainement pas les riches comme groupe. Car, ce qui est certain, c'est que c'étaient des capitalistes qui défendaient les droits des seigneurs ou encore leurs droits de seigneurs. C'est précisément la raison pour laquelle Francis Hincks, en 1854, préféra laisser renverser son gouvernement plutôt que d'accepter une loi d'abolition du régime seigneurial; il expliqua que la Province avait besoin d'emprunter de l'argent pour construire ses chemins de fer et que, si elle touchait aux droits des seigneurs, elle ne pourrait plus trouver à emprunter l'argent dont elle avait besoin. ${ }^{55} \mathrm{Et}$, à l'époque de l'abolition du régime seigneurial, les journaux anglais semblaient avoir changé de camp; à tel point qu'après le plaidoyer de Christopher Dunkin en faveur des seigneurs, quelqu'un écrivit dans La Minerve: "Il semble étrange à celui qui a lu, il y a vingt ans, dans cette même presse anglaise du Bas-Canada, des dénonciations extrêmement fortes contre le système féodal, et contre les abominables exactions des seigneurs, de voir l'attitude qu'elle prend aujourd'hui."

Si la masse demandait l'abolition, pourquoi n'a-t-elle obtenu qu'une réforme?

M. Ouellet soutient que les capitalistes, les petits bourgeois d'allégeance libérale, les "esprits réalistes de toutes teintes, politiques ou idéologiques", (ce qui semble représenter une partie importante de la population), menaient une campagne et

55 "Banquet donné au «London Tavern», en l'honneur de Lord Elgin, gouverneur général du Canada, La Minerve, 26-95 (25 avr. 1854).

56 Un Censitaire d'origine Britannique, "Tenure seigneuriale", La Minerve, 25-71 (31 mars 1853). 
"une campagne bien organisée en vue d'obtenir l'abolition" de la tenure seigneuriale. ${ }^{57}$ Puis, tout à coup, sans donner aucune explication sur la raison de ce revirement, il dit qu'on leur a accordé non pas ce qu'ils demandaient, c'est-à-dire l'abolition, mais seulement une réforme du régime seigneurial. ${ }^{58}$ La question qui jaillit spontanément et qui reste sans réponse, est la suivante: Si tout ce monde demandait l'abolition, s'ils ont mené "une campagne bien organisée", pourquoi ne leur a-t-on accordé qu'une réforme au lieu de l'abolition qu'ils demandaient? La question restera toujours sans réponse, car, comme nous l'avons expliqué ailleurs, ${ }^{59} c^{\prime} e s t$ l'inverse qui s'est passé: la majorité des censitaires demandaient une réforme du régime seigneurial et on leur a imposé une abolition de ce régime avec paiement d'une indemnité aux seigneurs.

\section{Y a-t-il eu augmentation de la valeur des terres des cultivateurs?}

Dans le domaine des choses étranges, relevons une déduction pour le moins étonnante de M. Ouellet. Il cite d'abord Ellice, qui soutient que, par suite de la tenure seigneuriale, "la valeur des propriétés dans les villes a éprouvé depuis quelques années une baisse formidable..." De cette affirmation, que conclut M. Ouellet ? "A ces deux points de vue, le régime seigneurial défavorise le petit propriétaire et le capitaliste. Les tensions démographiques en sont accrues, le cultivateur doit affecter des sommes considérables à l'acquisition d'une terre..." 60 Donc, de l'affirmation d'Ellice que la valeur des propriétés baisse dans les villes, M. Ouellet conclut que, dans les campagnes, la valeur des terres des cultivateurs augmente. Etrange déduction, à la vérité ! Si la tenure seigneuriale a fait subir une baisse à la valeur des propriétés dans les villes, pourquoi aurait-elle fait augmenter la valeur des propriétés dans les campagnes?

$57 \mathrm{~V}$. ci-devant, note 45.

58 Histoire économique, 467.

$59 \mathrm{G}$. Baillargeon, "La tenure seigneuriale a-t-elle été abolie par suite des plaintes des censitaires ?", Revue d'Histoire de l'Amérique française, 21-1, (juin 1967) : 76-78.

60 Histoire économique, 353. 


\section{Grand nombre de censitaires demandaient l'abolition sans indemnité}

M. Ouellet prétend que "personne n'envisage une abolition qui ne tiendrait pas compte des droits acquis". Et il ajoute: "L'idée d'indemnité est inhérente à toutes les options." "11 Mais, ce qui est le plus étrange, c'est que deux pages plus loin, ${ }^{62}$ il cite - en anglais - sans se rendre compte que cela contredit sa propre affirmation, le Rapport des Commissaires de 1843, qui dit exactement le contraire: "Nous pensons que les habitants d'origine Française n'ont pas un très-vif désir de changer la tenure de leurs terres... quoiqu'ils désirent beaucoup se libérer des charges Seigneuriales. Ils désirent se décharger des fardeaux qui pèsent sur eux avec le plus d'oppression, mais ils ne témoignent que rarement la volonté de donner un équivalent." ${ }^{63} \mathrm{Et}$, dans les lettres jointes à ce Rapport, certains correspondants font remarquer que les gens demandent à être libérés parce qu'ils croient que cela peut se faire sans qu'ils aient à payer, mais qu'ils ne demanderaient rien s'ils savaient combien il leur en coûtera pour se libérer. ${ }^{64}$ Un autre censitaire émettra plus tard la même opinion: "j'incline fort à croire que si les censitaires comprenaient clairement... tout ce qu'il leur faudra payer pour se défaire des seigneurs, ils aimeraient mieux les garder. La raison qui les fait consentir à l'abolition, est qu'on leur fait voir la chose tout en beau, et qu'on ne leur montre pas les sommes qu'ils auront à payer." 65 Un homme encore mieux placé pour

61 Histoire économique, 465.

62 Ibid., 467.

63 Notons que l'auteur cite ce passage en anglais alors qu'ailleurs il a cité la version française ! Voici comment il alterne: p. 463 réf. 44, et p. 464 réf. 48 , le rapport est cité en français. Mais, p. 465 réf. 50 , il est cité en anglais. Puis, treize lignes plus loin, p. 465 réf. 51, il est cité en français. Enfin, p. 467 réf. 56, il est de nouveau cité en anglais, pourtant le titre indiqué dans le renvoi est en français. De cette façon, seul un spécialiste qui connaît le document par cœur peut savoir que l'auteur cite toujours le même document.

64 No 39, "Réponses de W. Berczy", 14 nov. 1842, Rapport des Commissaires, 125.

65 "Pour la Minerve. Un mot sur la Tenure Seigneuriale", La Minerve, 26-92 (18 avril 1854). 
parler de ce sujet, Edward Gibbon Wakefield, ${ }^{66}$ un Britannique qui gérait la seigneurie de Beauharnois, le disait en ces termes:

Le fait est qu'il n'existe aucun désir parmi la population de l'une ou de l'autre origine, de se prévaloir de la faculté de commuer qui leur a été accordée par l'Acte des Tenures. Grand nombre de personnes des deux origines mais surtout les Censitaires Ecossais, font sonner bien haut leur désir de commuer; mais je n'en ai pas trouvé un seul qui, après lui avoir expliqué que le mot "commutation" signifie le rachat de la tenure pour une valeur équivalente, ne se soit écrié: “Oh ! ce n'est pas là ce que j'entends." La plupart des gens ne sont pas du tout disposés à racheter les lods et ventes futurs moyennant le paiement immédiat d'une somme fixe. (...)

J'ai remarqué qu'il existait une différence remarquable d'opinion parmi les deux origines au sujet de la commutation... Les Canadiens français reconnaissent plus volontiers que les gens d'origine britannique, qu'il serait injuste de changer cette tenure sans donner un équivalent au Seigneur. Ceux d'origine britannique, au contraire, semblent penser qu'il n'y a rien d'injuste à demander, sous le nom de commutation, une entière confiscation; car c'était là le cri des loyaux pendant l'élection du comté de Beauharnois. ${ }^{67}$

Bien plus, il y avait un curé qui recommandait l'abolition des droits lucratifs, lods et ventes et banalité en particulier, sans aucun dédommagement au seigneur: "Tout au plus on pourrait exiger d'eux [les censitaires] le capital de la rente... Il vaut mieux qu'un seigneur souffre que de laisser souffrir un peuple."68

${ }^{66}$ A. J. Harrop, The Amazing Caroer of Edward Gibbon Wakefield (Londres, 1928), 135; M. D. Law, éd., Chamber's Encyclopedia (New Ed., 15 vol., Londres, c. 1950), 14: 394; L. J. Burpee et A. G. Doughty, éd., Index and Dictionary of Canadian History, The Makers of Canada (éd. de Luxe), 21: 402; S. W. Wallace, The Dictionary of Canadian Biography (2e éd., Toronto, 1945), 2; S. Lee, éd., Dictionary of National Biography (68 vol., Londres, 1885-1904), 58 (1899).

67 No 69, "Opinion d'Edward Gibbon Wakefield", 2 sept. 1842, Rapport des Commissaires, 220.

68 No 21, "Réponses de M. Richard [Ricard]", 10 juin 1842, Rapport des Commissaires, 91. 
Cette idée d'abolition sans compensation s'était répandue à l'occasion des assemblées de comtés de 1836-37 et des "Troubles". Le notaire J.-B. Dupuy, de Laprairie, allait jusqu'à affirmer: "Je puis dire avec sûreté que si nous avons eu une insurrection dans ce pays, nous pouvons en attribuer la cause à la tenure seigneuriale." Quand je demandais aux habitants de la seigneurie de Laprairie et des seigneuries avoisinantes pourquoi ils se révoltaient, "ils me répondaient que c'était pour abattre les seigneurs, qui étaient leur ruine et que, par ce moyen, ils abattraient la tenure seigneuriale." " 9 Par ailleurs, un témoin des événements de Saint-Eustache rapporte que "les chefs (Chénier, Girod, Feriol Peltier, Hubert, etc.) promirent à leurs soldats l'abolition des dimes et des rentes seigneuriales". ${ }^{70}$ De même, l'abbé Ricard, curé de Saint-Marc, disait: “qu'en 1837 et '38, on ... faisait courir le peuple à la mort, en lui disant: vous ne paierez plus de rentes, vous ne paierez plus de lods". ${ }^{71}$ Enfin, on trouve une nouvelle preuve de l'ampleur prise par le mouvement visant à l'abolition sans compensation au cours de cette période dans le district de Montréal, dans la proclamation faite par Robert Nelson le 28 février 1838, alors qu'il annonçait que la tenure seigneuriale était abolie et que toute personne qui porterait les armes ou fournirait quelque assistance au Peuple dans sa lutte d'émancipation, serait "déchargée de toutes dettes on obligations réelles ou supposées, envers les seigneurs, pour arrérages en vertu de Droits seigneuriaux ci-devant existants". ${ }^{22}$ Ajoutons encore que beaucoup d'Anglais de Montréal avaient à plusieurs reprises demandé l'abolition de la tenure seigneuriale sans indemnité. ${ }^{73}$ En février 1840, l'Ami du Peuple écrivait: "les abolitionnistes de la tenure féodale (sans indemnité bien enten-

${ }^{69}$ No 23, "Réponses de J.-B. Dupuy", 12 juill. 1842, Rapport des Commissaires, 94.

70 Montreal Herald, 32-12 (28 janv. 1840); tiré du Journal Historique des evenemens arrivés à St. Eustache, par un témoin oculaire.

71 No 21, "Réponses de M. Richard [Ricard]", 10 juin 1842, Rapport des Commissaires, 91.

72 M. Brunet, G. Frégault, M. Trudel, éd., Histoire dw Canada par les textes, 159.

73 "Pétition des habitants britanniques et autres résidant dans la ville et les environs de Montréal", 21 juill. 1838, APC, Durham 5-1, 605. 
$d u$ ) après avoir tenté ..." et le Canadien y faisait écho pour dire qu'il fallait rejeter toute idée de spoliation et qu'il faudrait payer pour se débarrasser de la tenure seigneuriale. ${ }^{74}$ Quand l'Avenir parla de décharger les censitaires des droits seigneuriaux, il prit la peine de spécifier que c'était "à la condition de les affranchir en indemnisant les seigneurs". ${ }^{75}$ Quelque temps après, c'était le Sherbrooke Journal qui jugeait à propos d'éclairer ses lecteurs sur ce sujet; le Montreal Transcript reproduisit l'article:

Le malheur est que Censitaires et autres qui se sont montrés opposés au système, procèdent comme si tous ses mauvais effets étaient imputables aux Seigneurs, et comme si c'était un vol de la part des Seigneurs que de réclamer leurs droits légaux, mais que ce n'en était pas un pour les censitaires que de les dépouiller de ces droits, sans compensation.

Le revenu du Seigneur provenant des lods et ventes, du droit de banalité, du droit de rachat et d'autres sources semblables, est protégé par la loi autant que celui de n'importe quel fermier de ce district. (...) Si la féodalité... entrave... la prospérité de tous, on doit jeter la féodalité par dessus bord. - Nous serons toujours prêt à lui donner un coup d'épaule, mais en même temps, qu'on se rappelle que si nous y sommes opposé, nous ne pouvons pas, en justice, demander qu'un bénéfice public soit obtenu aux dépens et aux frais d'une seule classe. ${ }^{76}$

En 1848, la Minerve revenait sur le sujet: "on prétend que ce désir... [de l'abolition du régime seigneurial] doit être attribué à l'espérance que des chercheurs de popularité ont fait naître dans l'esprit des censitaires que cette abolition pouvait être obtenue sans compensation aucune pour les seigneurs... (...) il est désirable que la législature... s'empresse de déclarer que si l'abolition de la tenure seigneuriale doit être décrétée, elle ne peut l'être et ne le sera qu'à la condition d'une juste indemnité de la part du censitaire envers son seigneur". ${ }^{77}$ Au cours de la

74 "De l'Ami du Peuple", Le Canadien, 9-107 (10 févr. 1840).

75 F. André-Julien, Les textes essentiels du journal "l'Avenir" 18471858 (Manuscrit, Univ. de Montréal), 166.

76 Montreal Transcript, 4-129, (27 févr. 1840), 514.

77 “Tenure seigneuriale", La Minerve, 21-14 (26 oct. 1848). 
session de 1850, Louis-Hippolyte LaFontaine jugea nécessaire de déclarer devant l'Assemblée législative: " $i l$ y a actuellement des gens qui pensent que la tenure seigneuriale doit être abolie purement et simplement sans indemniser les seigneurs... Mais plus cette opinion est forte, plus la chambre doit se prononcer avec fermeté pour montrer aux parties intéressées qu'il ne peut y avoir de commutation sans indemnité au seigneur pour toute la valeur de ses droits." 78 Après quoi LaFontaine fit adopter par la Chambre la résolution suivante: "la dite commutation ne peut avoir lieu qu'au moyen d'une indemnité suffisante en faveur de tous ceux dont les justes droits seront lésés en l'effectuant." 79 Malgré cette prise de position officielle, l'idée continua à faire son chemin, si bien qu'en 1853 Joseph Cauchon se crut obligé de dire en Chambre:

C'est avec chagrin que j'ai entendu des gens parfaitement honnêtes par ailleurs, dire qu'ils sont prêts à faire main basse sur les droits seigneuriaux. L'instinct de l'intérêt l'emporte sur la raison. (...) je n'admettrai jamais que l'on puisse spolier les seigneurs de leurs droits acquis, quelle que puisse être la force de l'opinion publique; si on enlève des droits pour utilité publique, il faut indemniser. ${ }^{80}$

Il faut donc reconnaître que, contrairement à ce qu'affirme M. Ouellet, un grand nombre de censitaires, et pas seulement de langue française, réclamaient l'abolition pure et simple des droits seigneuriaux sans indemnité aux seigneurs.

\section{Conclusion}

Ayant personnellement encouru le risque de poursuites judiciaires pour prouver précisément que le régime seigneurial avait vraiment été aboli, ${ }^{81}$ nous ne pouvions pas nous permettre de laisser passer les affirmations contraires. Il y a des milliers 1850).

${ }^{78}$ "Chambre d'Assemblée", 14 juin 1860, La Minerve, 22-83 (20 juin

79 Journaux de l'Assemblée législative de la province du Canada, 9 (1850), 96.

80 "Tenure seigneuriale", La Minerve, 25-85 (10 mai 1853).

$81 \mathrm{G}$. Baillargeon, La survivance du régime seigneurial à Montréal, 164. 
et des milliers de dollars en jeu dans cette discussion; ${ }^{82}$ une poursuite judiciaire s'impose pour faire toute la lumière sur la question; ${ }^{83}$ l'heure n'est pas aux affirmations gratuites. Le plus cocasse dans toute cette affaire, c'est que $M$. Ouellet, qui blâme les "clercs" ${ }^{84}$ d'avoir fait des efforts pour maintenir le régime seigneurial, est passé sans s'en rendre compte dans leur camp, en soutenant que le régime seigneurial n'a pas été aboli ${ }^{85}-$ exactement ce que soutiennent les "clercs" ex-seigneurs de Montréal !

Collège militaire

\section{Georges BAILLARGeON}

Saint-Jean, Qué.

82 Ibid., 152.

${ }^{83} \mathrm{~J}$. Boucher, Revue d'Histoire de l'Amérique fransaise, 22-1 (juin 1968) : 128 .

84 Histoire économique, 462.

85 Ibid., 467. 